\title{
Evaluación de la Percepción del Proceso de Enseñanza y Aprendizaje de un Curso de Morfología Humana por parte de Estudiantes de un Programa para Talentos Académicos
}

\author{
Evaluation of the Teaching and Learning Process in a Human Morphology Course by Students \\ from an Academic Talents Program
}

\author{
Rossy Olivares $^{* \text {,*** }}$; Ricardo Henríquez*; Cristina Simpson ${ }^{* * *}$; Octavio Binvignat ${ }^{* * * * *}$; \\ Marcela González $^{* * * * * *}$; Leonor Conejeros ${ }^{* * * * * * *}$; Cristian Merino ${ }^{* * * * * * *}$ \& Pablo Lizana Arce*
}

OLIVARES, R.; HENRÍQUEZ, R.; SIMPSON, C.; BINVIGNAT, O.; GONZÁLEZ, M.; CONEJEROS, L.; MERINO, C. \& LIZANA, P. A. Evaluación de la percepción del proceso de enseñanza y aprendizaje de un curso de morfología humana por parte de estudiantes de un programa para talentos académicos. Int. J. Morphol., 32(1):141-146, 2014.

RESUMEN: La alta capacidad intelectual se considera una manifestación diferencial del desarrollo cognitivo, siendo la estimulación temprana y sistemática favorecedores de su expresión y desarrollo. Es por ello, que otorgar oportunidades educativas enriquecidas es central para los los niños y niñas con talentos académicos. Esta investigación evalúa la percepción de los estudiantes frente a una metodología de enseñanza teórico-práctica en un curso dirigido a talentos académicos de enseñanza media (n=17) en el área de morfología humana. Los estudiantes formaron parte de un programa universitario de enriquecimiento extracurricular. Al final del periodo los estudiantes evaluaron los cursos mediante una encuesta referida a: i) el curso ii) el profesor, y iii) autoevaluación. Los resultados de la comparación de las evaluaciones realizadas por los estudiantes que participaron en dicho curso versus estudiantes de otros cursos $(\mathrm{n}=128)$ muestran diferencias significativas $(\mathrm{p}<0,05)$. El talento académico que presenta este tipo de estudiantes complementado con la metodología de trabajo aplicada, permite que avancen secuencialmente y acorde a sus capacidades, en un ambiente de trabajo propicio para tal desarrollo. Además, este tipo de intervenciones fomenta el estudio de la morfología humana en estudiantes con talento académico.

PALABRAS CLAVE: Talento Académico; Proceso de Enseñanza y Aprendizaje; Morfología humana.

\section{INTRODUCCION}

Durante el crecimiento de todo individuo se produce no sólo un desarrollo físico, social y psicológico sino también un desarrollo cognitivo, el cual se origina tras una interacción entre la herencia genética, el entorno y el constructivismo neural de dicho individuo (Sastre-Riba, 2008).

La alta capacidad intelectual que poseen ciertas personas es una manifestación diferencial del desarrollo cognitivo en donde prevalece esta capacidad si ha sido potenciada y desarrollada sistemáticamente. Denominaremos a esta alta capacidad como dotación, que se define como las aptitudes naturales sobresalientes (Gagné, 2008).

Aún cuando se reconoce un importante componente hereditario, se asume que el talento requiere de un contexto adecuado, que fomente y desafíe a la persona a desarrollar sus potencialidades (Arancibia, 2009). A lo anterior se le

\footnotetext{
* Laboratorio de Técnicas Anatómicas, Instituto de Biología, Facultad de Ciencias, Pontificia Universidad Católica de Valparaíso, Valparaíso, Chile.

** Programa de Magíster en Ciencias, Mención Morfología, Universidad de La Frontera, Temuco, Chile.

*** Instituto de Estadística, Pontificia Universidad Católica de Valparaíso, Valparaíso, Chile.

***** Facultad de Ciencias de la Salud. Universidad Autónoma de Chile, Talca, Chile.

****** Programa BETA-PUCV, Buenos Estudiantes con Talentos Académicos - Pontificia Universidad Católica de Valparaíso, Valparaíso, Chile.

******** Escuela de Pedagogía, Pontificia Universidad Católica de Valparaíso, Valparaíso, Chile.

******** Laboratorio de Didáctica, Instituto de Química, Pontificia Universidad Católica de Valparaíso, Valparaíso, Chile.
} 
OLIVARES, R.; HENRÍQUEZ, R.; SIMPSON, C.; BINVIGNAT, O.; GONZÁLEZ, M.; CONEJEROS, L.; MERINO, C. \& LIZANA, P. A. Evaluación de la percepción del proceso de enseñanza y aprendizaje de un curso de morfología humana por parte de estudiantes de un programa para talentos académicos. Int. J. Morphol., 32(1):141-146, 2014.

conoce como catalizadores, los que pueden ser de tipo ambiental e intrapersonal. Para Gagné (2008) éstos incidirán en el tránsito de la dotación o habilidades naturales no entrenadas al talento, siendo este último la expresión manifiesta de la dotación. En este sentido, es imprescindible que los estudiantes con alta capacidad cuenten con un entorno significativo, experiencias oportunas y adecuadas de aprendizaje que les permitan desarrollar dicho potencial. En Chile, Conejeros et al. (2012) indican que se ha optado por trabajar con un grupo de estudiantes que presentan altas capacidades en la dimensión intelectual, denominados Talentos Académicos, término que según indica Arancibia et al. (2010), se encuentra referido a niños y jóvenes que demuestran habilidades intelectuales generales o específicas que los sitúan en el 10\% superior del promedio de sus pares. Estas habilidades se expresarían a través de las áreas que aborda el currículum científico-humanista de la formación escolar, es decir: matemáticas, humanidades, ciencias sociales y ciencias básicas.

Este grupo de estudiantes presenta características diferenciales en su desarrollo socio-emocional, tales como: a) presencia de mayor sensibilidad e intensidad emocional (Piechowski, 1997), b) una aptitud acentuada para comprender y transformar las percepciones en experiencias intelectuales y emotivas (Roeper, 1982), c) perfeccionismo y necesidad de precisión (Schuler, 2002) y finalmente d) asincronía, definida como un desfase en el desarrollo de las áreas cognitiva, social y emocional (Silverman, 1993).

Diversos estudios han demostrado que estos alumnos poseen predisposición a presentar problemas sociales y emocionales (Alencar, 2007) vinculados mayoritariamente a desmotivación y frustración cuando se ven enfrentados a programas académicos repetitivos, monótonos, con poco desafío y climas de aula poco favorables a la expresión de su potencial (Alencar, 2008). Estos riesgos son corroborados por Reis \& Renzulli (2004), quienes basándose en una revisión reciente de la literatura en el ámbito socio-emocional señalan que los estudiantes con altas capacidades podrían enfrentar situaciones de riesgo para su desarrollo emocional al no encontrar ambientes educacionales que tomen en cuenta su ritmo de aprendizaje, su nivel de desarrollo intelectual y disponer de actividades de aprendizaje desafiantes.

Por lo anterior, se describe la importancia del ambiente de trabajo en donde se desenvuelven estos estudiantes, potenciando sus talentos, permitiendo su desarrollo sistemático, creando instancias en donde éstos compartan lo que saben y se comuniquen unos con otros (Gagné, 2011). En este escenario el docente cobra importancia: "los estudiantes con altas capacidades requieren de alguien que pueda acompañarlos en la búsqueda de respuestas y que se preocupe por conocerlos y apoyarlos de manera genuina" (Conejeros-Solar et al., 2013). Contar con altas expectativas de los estudiantes cobra relevancia, pues dicha influencia puede afectar favorablemente en su rendimiento. Lo anterior denominado "Efecto Pigmalión" fue introducido por Rosenthal \& Jacobson el año 1968 y se define principalmente como las altas expectativas que una persona pueda poseer sobre otra pueden influir favorablemente en su rendimiento (Alencar, 2007). Así, el docente es un ente fundamental en el desarrollo, tanto cognitivo como emocional de un estudiante con talento académico.

A nivel mundial, diversos organismos e instituciones del ámbito privado y público han desarrollado variadas propuestas educativas para estudiantes con talentos académicos. En nuestro país, principalmente las universidades se han ocupado de esta tarea e.g. Programa de estudios y desarrollo de talentos PENTA UC / Pontificia Universidad Católica de Chile, Santiago, Región Metropolitana - Programa talentos UdeC / Universidad de Concepción, Concepción, Región del Bío Bío - Programa educacional para niños, niñas y jóvenes con talentos académicos de la Universidad de La Frontera / Universidad de La Frontera, Temuco, Región de La Araucanía, utilizando una modalidad extracurricular. Esto principalmente responde a la preocupación por no desarraigar al estudiante de su medio escolar y social natural (Arancibia, 2009).

Una de las instituciones universitarias que brinda este servicio en nuestro país, específicamente en la región de Valparaíso, es la Pontificia Universidad Católica de Valparaíso con el programa extracurricular Buenos Estudiantes con Talento Académico de la Pontificia Universidad Católica de Valparaíso (BETA-PUCV). Dicho programa comenzó el año 2006 y aspira principalmente a fortalecer y enriquecer las potencialidades de los alumnos/ as con talentos académicos, así como también a formar personas creativas, críticas y propositivas, motivadas por el conocimiento y dispuestas a ejercer su responsabilidad social a través de cursos y talleres en distintas áreas complementarios a la educación escolar (BETA-PUCV, 2013).

A partir de lo anterior, se investigó acerca de las percepciones que tienen los estudiantes con talento académico sobre un curso de morfología humana en relación con los siguientes puntos: en qué grado las actividades diseñadas fomentan su aprendizaje en profundidad de la disciplina, sobre el docente y su propio desempeño en el curso, evidenciando si la metodología aplicada les permite potenciar tanto su desarrollo cognitivo como también su desarrollo social y emocional. 
OLIVARES, R.; HENRÍQUEZ, R.; SIMPSON, C.; BINVIGNAT, O.; GONZÁLEZ, M.; CONEJEROS, L.; MERINO, C. \& LIZANA, P. A. Evaluación de la percepción del proceso de enseñanza y aprendizaje de un curso de morfología humana por parte de estudiantes de un programa para talentos académicos. Int. J. Morphol., 32(1):141-146, 2014.

\section{MATERIAL Y MÉTODO}

Descripción de la muestra. El estudio fue realizado en el laboratorio de ciencias de la Facultad de Ciencias del Mar de la PUCV. Los participantes de esta investigación fueron 17 estudiantes de una edad promedio de $16 \pm 0,93$ de ambos géneros y con 2,75 $\pm 2,13$ años en el programa BETA-PUCV. Todos los estudiantes cursaron la asignatura "Morfología Humana", impartida durante el segundo semestre 2012 (Tabla I). Los sujetos pertenecían a la Región de Valparaíso (Valparaíso 41,67\%; Viña del Mar 25\%; Quilpué 20,83\%; Quillota 8,33\%; Villa Alemana 4,17\%).

Actividades de aprendizaje (AA)

AA1 / Disecciones

AA2 / Observación de muestras histológicas

AA3 / Confección de modelos anatómicos

AA4 / Desarrollo de técnicas anatómicas en el curso de Morfología Humana.
Descripción de las actividades. El método aplicado durante el curso incluyó estrategias activo-participativas que comprendieron: clases interactivas (aprendizaje basado en problemas, estudio de casos), pasos prácticos (disecciones, observación de muestras histológicas), confección de modelos tridimensionales, desarrollo de técnicas anatómicas y un refuerzo continuo a través del Aula Virtual de Anatomía Humana. Como curso de morfología se abordaron temáticas de histología, embriología y anatomía humana (Fig. 1).

Descripción de los instrumentos. Para evaluar la percepción de los estudiantes se aplicó al final del curso un cuestionario conformado por un grupo de aseveraciones y de respuestas tipo escala Likert (muy de acuerdo, de acuerdo, en parte de acuerdo, en desacuerdo), el cual se encontraba dividido en tres secciones (Tabla II).

Análisis Estadístico. Los análisis correspondieron al número de respuestas Muy de Acuerdo (MA) de la escala Likert aplicada. Las distintas proposiciones de la evaluación final del curso de "Morfología Humana" (n= 17) fueron comparados con la proporción de respuestas MA que muestra el resto de los cursos BETA-PUCV (la cual fue usada como patrón, $n=128$ ). El análisis se realizó calculando el valor-p mediante la distribución binomial, considerando significativo un valor $\mathrm{p}<0,05$.

\section{RESULTADOS}

En las Tablas III, IV y V se presentan los análisis comparativos realizados frente a las preguntas que conforman las tres secciones de la encuesta (comparación estudiantes de Morfología Humana versus resto de los cursos BETA-PUCV). Los resultados obtenidos muestran en su mayoría que los estudiantes tienen una percepción favorable en este tipo de curso.

\section{DISCUSIÓN}

Este estudio se basa en la comparación de la aplicación de una metodología teórica-práctica, centrada en el estudiante y desarrollada en tres ambientes de trabajo (teó- 
OLIVARES, R.; HENRÍQUEZ, R.; SIMPSON, C.; BINVIGNAT, O.; GONZÁLEZ, M.; CONEJEROS, L.; MERINO, C. \& LIZANA, P. A. Evaluación de la percepción del proceso de enseñanza y aprendizaje de un curso de morfología humana por parte de estudiantes de un programa para talentos académicos. Int. J. Morphol., 32(1):141-146, 2014.

Tabla I. Características de la muestra.

\begin{tabular}{cccccccc}
\hline \multicolumn{2}{c}{ Sexo } & \multicolumn{3}{c}{ Curso - Grado } & \multicolumn{3}{c}{ Procedencia } \\
\hline $\mathrm{M}$ & $\mathrm{F}$ & 10 & 11 & 12 & MU & PS & PSS \\
33,35 & 66,65 & 41,67 & 45,83 & 12,50 & 66,67 & 29,17 & 4,17 \\
\hline
\end{tabular}

Nota: datos en porcentaje. $M=$ masculino, $\mathrm{F}=$ femenino, $\mathrm{MU}=$ municipalizado, $\mathrm{PS}=$ particular con subvención, $\mathrm{PSS}=$ particular sin subvención.

Tabla II. Descripción de secciones e ítems del instrumento.

\begin{tabular}{|c|c|c|}
\hline Secciones & Número de ítems & Ejemplo de pregunta \\
\hline $\begin{array}{l}\text { I. Percepciones del estudiante sobre el } \\
\text { curso. }\end{array}$ & $1,2,3$ & $\begin{array}{l}\text { Fomenta el desarrollo del lenguaje a ni vel de recepción } \\
\text { argumentación y producción de discursos orales y/o escritos. }\end{array}$ \\
\hline $\begin{array}{l}\text { II. Percepciones del estudiante sobre el } \\
\text { Prof esor. }\end{array}$ & $\begin{array}{l}4,5,6,7,8,9,10,11 \\
12\end{array}$ & $\begin{array}{l}\text { Comunica el sentido de las actividades a realizar en } \\
\text { relación a las competencias definidas al interior del curso. }\end{array}$ \\
\hline III. Autoevaluación del estudiante. & $13,14,15,16,17$ & $\begin{array}{l}\text { Presentó una buena disposición hacia el curso y las actividades } \\
\text { propuestas. }\end{array}$ \\
\hline
\end{tabular}

Tabla III. Evaluación de la percepción de los estudiantes sobre los cursos.

\begin{tabular}{lccc}
\hline Afirmación & $\begin{array}{c}\text { Curso } \\
\text { Morfología\% }\end{array}$ & $\begin{array}{c}\text { Resto de cursos } \\
\text { BETA\% }\end{array}$ & $\begin{array}{c}\text { Curso Morfología/resto } \\
\text { de cursos Beta Valor p }\end{array}$ \\
\hline $\begin{array}{l}\text { 1. Se prioriza la profundización por sobre la amplitud de los } \\
\text { contenidos. }\end{array}$ & 94,11 & 75,78 & $0,008^{*}$ \\
$\begin{array}{l}\text { 2. Fomenta el d esarrollo del lenguaje a ni vel de recepción, } \\
\text { argumentación y producción de discursos orales y/o escritos. }\end{array}$ & 76,47 & 81,25 & 0,601 \\
3. Cumplió las expectativas que tenia. & 88,23 & 67,18 & $0,010^{*}$ \\
\hline
\end{tabular}

Tabla IV. Evaluación de la percepción de los estudiantes sobre el profesor.

\begin{tabular}{|c|c|c|c|}
\hline Afirmación & $\begin{array}{c}\text { Curso } \\
\text { Morfología\% }\end{array}$ & $\begin{array}{l}\text { Resto de cursos } \\
\text { BETA\% }\end{array}$ & $\begin{array}{l}\text { Curso Morfología/re sto } \\
\text { de cursos Beta Valor p }\end{array}$ \\
\hline 1. Estimula la participación de todos los estudiantes. & 94,11 & 80,46 & $0,024 *$ \\
\hline $\begin{array}{l}\text { 2. Se crea un clima de relaciones interpersonales empáticas con sus } \\
\text { estudiantes y entre ellos. }\end{array}$ & 88,23 & 74,21 & $0,043 *$ \\
\hline $\begin{array}{l}\text { 3. Desarrolla en los alumnos la capacidad de adaptación y de } \\
\text { autoaprendizaje. }\end{array}$ & 100 & 75,78 & $0,000 *$ \\
\hline $\begin{array}{l}\text { 4. Las estrategias que implementa desarrollan compromiso por el } \\
\text { trabajo, confianza en sí mismo y sentido de logro creativo. }\end{array}$ & 88,23 & 71,87 & $0,027 *$ \\
\hline $\begin{array}{l}\text { 5. Transmite una moti vación positiva por el aprendizaje, la } \\
\text { indagación y la búsqueda, más allá de lo trabajado en clases. }\end{array}$ & 94,11 & 75,87 & $0,008^{*}$ \\
\hline $\begin{array}{l}\text { 6. Comunica el sentido de las actividades a realizar en relación a las } \\
\text { competencias definidas al interior del curso. }\end{array}$ & 82,35 & 77,34 & 0,223 \\
\hline $\begin{array}{l}\text { 7. Evalúa cualitativamente y re troalimenta los procesos de } \\
\text { aprendizaje. }\end{array}$ & 94,11 & 81,25 & $0,029^{*}$ \\
\hline $\begin{array}{l}\text { 8. Invita a los alumnos a compartir sus propios conocimientos y } \\
\text { aprendizajes con otros/as. }\end{array}$ & 94,11 & 82,81 & $0,040^{*}$ \\
\hline 9. Demuestra entusiasmo por lo que enseña. & 94,11 & 88,28 & 0,120 \\
\hline
\end{tabular}

* Indica diferencias significativas $\mathrm{p}<0,05$.

Tabla V. Autoevaluación del estudiante sobre su desempeño en los cursos.

\begin{tabular}{|c|c|c|c|}
\hline Afirmación & $\begin{array}{c}\text { Curso } \\
\text { Morfología \% }\end{array}$ & $\begin{array}{l}\text { Resto de cursos } \\
\text { BETA\% }\end{array}$ & $\begin{array}{l}\text { Curso neurociencia/ resto } \\
\text { de cursos Beta Valor } p\end{array}$ \\
\hline Me esfuerzo por alcanzar las metas propuestas por el curso & 88,23 & 60,15 & $0,002 *$ \\
\hline $\begin{array}{l}\text { Presento una buena disposición hacia el curso yl as } \\
\text { actividades propuestas. }\end{array}$ & 88,23 & 60,93 & $0,002 *$ \\
\hline Me siento comprometido/a con el programa & 94,11 & 75,78 & $0,008 *$ \\
\hline $\begin{array}{l}\text { Cumplo con la normativa del programa (asistencia, } \\
\text { justificación de inasistencia, puntualidad, etc,). }\end{array}$ & 76,47 & 43,75 & $0,001 *$ \\
\hline
\end{tabular}

* Indica diferencias significativas $\mathrm{p}<0,05$. 
OLIVARES, R.; HENRÍQUEZ, R.; SIMPSON, C.; BINVIGNAT, O.; GONZÁLEZ, M.; CONEJEROS, L.; MERINO, C. \& LIZANA, P. A. Evaluación de la percepción del proceso de enseñanza y aprendizaje de un curso de morfología humana por parte de estudiantes de un programa para talentos académicos. Int. J. Morphol., 32(I):141-146, 2014.

rico, práctico y virtual) (Lizana et al., 2008) en un curso de Morfología Humana para estudiantes con talento académico contrastada con las aplicadas en el resto de los cursos BETA-PUCV, siendo aceptada favorablemente por los estudiantes. Esta percepción favorable de los estudiantes con talento académico ya había sido replicada en estudiantes de enseñanza básica (Lizana et al., 2008) y enseñanza media en un curso de neuroanatomía funcional (Lizana et al., 2010). La reflexión cuidadosa de estas instancias de evaluación por parte los estudiantes con talento académico son relevantes para mejorar las estrategias de aprendizaje en próximas oportunidades de intervención (Van Tassel-Baska, 2002).

Los resultados sobre el cumplimiento de las expectativas de los estudiantes con respecto al curso de Morfología y su favorable percepción acerca de la profundización de contenidos por sobre su cantidad puede tener directa relación con las competencias del profesor encargado que fueron reportadas por los estudiantes y coinciden con los aspectos que se esperan de un profesor de estudiantes talentosos y que fueron trabajados en cada una de las actividades planteadas para los estudiantes:incita constantemente a sus estudiantes a querer saber y a aprender más con contenidos desafiantes (Croft, 2003), reconoce sus características motivacionales, emocionales y cognitivas (Preckel et al., 2008), es un ente motivador dentro y fuera del aula, favorece el desarrollo cognitivo (pensamiento crítico, capacidad de análisis, creatividad, etc.) y posee altas expectativas académicas sobre sus estudiantes y de desarrollo personal, tanto de su grupo curso como de sus estudiantes en forma individual (Alencar, 2007).

En cuanto al ítem autoevaluación del estudiante, las cifras presentan diferencias significativas con el resto de los cursos, aspecto que es diferente a resultados anteriores (Lizana et al., 2010) donde no hubo diferencias significativas. Sin embargo, sí coincide con presentar los más bajos porcentajes respecto a los otros ítems evaluados, tendencia que se debe a su alta capacidad de autocrítica (Kingore, 2003) y alto grado de perfeccionismo (Schuler, 2000).

Se concluye que la metodología que integra las clases interactivas, la realización de trabajos prácticos que incluyen disecciones, confección de modelos tridimensionales y preparación de técnicas anatómicas, además de un refuerzo a través del Aula Virtual de Anatomía Humana fomenta el desarrollo y potencialidad del estudiante, siendo propicio para alumnos con talento académico ya que propone mayor variedad de estrategias de aprendizaje y flexibilidad en el desarrollo curricular, puesto que se ha demostrado que este tipo de estudiante, para aprender contenidos de alto nivel, requieren el desarrollo de diferentes tipos y niveles de habilidad (Van Tassel-Baska, 2002) con el fin de permitir que cada estudiante avance secuencialmente dependiendo de sus capacidades, se introduzca en un ambiente de trabajo propicio para tal desarrollo y además descubra a través de la experiencia directa la morfología humana.

\section{AGRADECIMIENTOS}

A la Dirección de Investigación, Vice-rectoría de Investigación y Estudios Avanzados, Instituto de Biología de la Facultad de Ciencias, a la Dirección de Desarrollo Curricular y Formativo y al programa de enriquecimiento curricular BETA-PUCV. Pontificia Universidad Católica de Valparaíso, Chile, por el apoyo y financiamiento.

OLIVARES, R.; HENRÍQUEZ, R.; SIMPSON, C.; BINVIGNAT, O.; GONZÁlEZ, M.; CONEJEROS, L.; MERINO, C. \& LIZANA, P.A. Evaluation of the teaching and learning process in a human morphology course by students from an academic talents program. Int. J. Morphol., 32(1):141-146, 2014.

SUMMARY: High intellectual capacity is considered to be a differential characteristic of cognitive development, noting that early and systematic stimuli encourages its expression and advancement. It is therefore, essential for academically talented children to be given enriched educational opportunities. This research project evaluates the students perceptions in a theoretical-practical teaching methodology in a course directed at high school academic talents in the area of human morphology. The students were part of a university program for extra-curricular enrichment. At the end of the period, the students assessed the courses by means of a survey referred to: i) the course ii) the professor and iii) self-evaluation. The results taken from the comparisons of the evaluations of the students participating in the course versus the students from other courses $(n=128)$ showed significant differences $(p<0.05)$. The academic talent represented by this type of student, complemented with the applied work methodology, allows the students to sequentially progress according to their capacities in an appropriate work environment for their development. Furthermore, it also encourages human morphology study in students who are academically talented.

KEY WORDS: Academic talent; Teaching and learning process; Human morphology.

\section{REFERENCIAS BIBLIOGRÁFICAS}

Alencar, E. Dificultades socio-emocionales del alumno con altas habilidades. Rev. Psicol., 26(1):43-62, 2008

Alencar, E. M. L. S. Caracteristicas socio-emocionais do superdotado: Questoes atuais. Psicol. Estud., 12(2):371-8, 2007. 
OLIVARES, R.; HENRÍQUEZ, R.; SIMPSON, C.; BINVIGNAT, O.; GONZÁLEZ, M.; CONEJEROS, L.; MERINO, C. \& LIZANA, P. A. Evaluación de la percepción del proceso de enseñanza y aprendizaje de un curso de morfología humana por parte de estudiantes de un programa para talentos académicos. Int. J. Morphol., 32(1):141-146, 2014.

Arancibia, V.; Boyanova, D. \& Moreno, M. Gifted education in Chile - the Program PENTA UC, Universidad Católica de Chile. Center for the Study and Development of Talent PENTA UC, 2010. Available in: http://www.pentauc.cl

Arancibia, V. La educación de alumnos con talentos: una deuda y una oportunidad para Chile. Centro de estudios y desarrollo de talentos, Pontificia Universidad Católica de Chile, 2009.

BETA-PUCV. Programa Buenos estudiantes con Talento Académico. Chile, 2013. Disponible en: http://www.programabeta.cl/

Conejeros-Solar, M. L.; Gómez-Arizaga, M. P. \& Donoso-Osorio, E. Perfil docente para alumnos/as con altas capacidades. Magis, 5(11):393-411, 2013

Conejeros, M. L.; Cáceres, P. \& Riveros, A. Educación de Talentos Académicos en Chile: Una década de aprendizajes e investigación. En: Catalán, J. (Ed.). Investigación orientada al cambio en psicología educacional. La Serena, Editorial Universidad de La Serena, 2012.

Croft, L. J. Teachers of the gifted: Gifted teachers. En: Colangelo, N. \& Davis, G. (Eds.). Handbook of gifted education. New York, Allyn and Bacon, 2003.

Gagné, F. Construyendo el talento a partir de la dotación: Breve revisión del MDDT 2.01. Montreal, Universidad de Quebec, 2008.

Gagné, F. Academic talent development and the equity issue in gifted education. Talent Development \& Excellence, 3(1):3$22,2011$.

Kingore, B. High achiever, gifted learner, creative thinker. Understanding our gifted, 15(3):3-5, 2003. Disponible en: h t t p : / / w w w. gif ted te a m.org/pd f/links / Gifted_vx_High_Acheiver_vx_Creative_Thinker.pdf

Lizana, P.; Almagià, A.; Barraza, F. \& Rodriguez, F. Diseño de un modelo neuroeducativo para la enseñanza y aprendizaje de las ciencias morfológicas. Int. J. Morphol., 26(1):170-226, 2008.

Lizana, P.; Almagia A.; Simpson, C.; Binvignat, O.; Henríquez, R.; Gómez, M.; González M \& Conejeros, M. Evaluación de la Enseñanza y Aprendizaje por Parte de Estudiantes de un Programa de Talentos Académicos (BETA-PUCV) Frente a un Curso de Neuroanatomía Funcional. Int. J. Morphol., 28(4):1245-9, 2010.

Piechowski, M. Emotional giftedness: The measure of intrapersonal intelligence: En: Colangelo, N. \& Davis, G. (Eds.). Handbook of Gifted Education. Boston, Allyn and Bacon, 1997.

Preckel, F.; Goetz, T.; Pekrun, R. \& Kleine, M. Gender Differences in Gifted and Average Ability Students: Comparing Girls' and Boys' Achievement, Self-Concept, Interest, and motivation in mathematics. Gifted Child Quart., 52(2):146-59, 2008.
Reis, S, \& Renzulli, J. Current research on the social and emotional development of gifted and talented students: Good news and future possibilities. Psychol. Schools, 41(1):119-30, 2004.

Roeper, A. How the gifted cope with their emotions. Roeper Rev., $5(2): 21-4,1982$.

Sastre-Riba, S. Niños con altas capacidades y su funcionamiento cognitivo diferencial. Rev. Neurol., 46(Supl. 1):S11-6, 2008.

Schuler, P. Perfectionism in gifted children and adolescents. En: Neihart, M.; Reis, S.; Robinson, N. \& Moon, S. (Eds.). The social and emotional development of gifted childen. Waco, Prufrock Press, 2002.

Silverman, L. K. Counseling the gifted and talented. Denver, Love Publishing Co., 1993.

Van Tassel-Baska, J. Planning effective curriculum experiences for gifted learners. Understanding Our Gifted, 15(1):6-8, 2002. Disponible en: http://www.davidsongifted.org/db/ Articles_id_10278.aspx

\section{Dirección para Correspondencia:}

Dr. Pablo Lizana Arce.

Laboratorio de Técnicas Anatómicas

Instituto de Biología

Pontificia Universidad Católica de Valparaíso. Chile.

2373223 Valparaíso

CHILE

Email: pablo.lizana@ucv.cl

Recibido : 09-10-2013

Aceptado: 17-12-2013 